\title{
COASTAL EROSION REVEALS A POTENTIALLY UNIQUE OLIGOCENE AND POSSIBLE PERIGLACIAL SEQUENCE AT PRESENT-DAY SEA LEVEL IN PORT DAVEY, REMOTE SOUTH-WEST TASMANIA
}

\author{
by Mike Macphail, Chris Sharples, David Bowman, Sam Wood \& Simon Haberle
}

(with two text-figures, five plates and two tables)

\begin{abstract}
Macphail, M., Sharples, C., Bowman, D., Wood, S. \& Haberle, S. 2014 (19:xii): Coastal erosion reveals a potentially unique Oligocene and possible periglacial sequence at present-day sea level in Port Davey, remote South-West Tasmania. Papers and Proceedings of the Royal Society of Tasmania 148: 43-59. https://doi.org/10.26749/rstpp.148.43 ISSN 0080-4703 Department of Archaeology \& Natural History, College of Asia and the Pacific, Australian National University, Canberra, ACT 0200, Australia $\left(\mathrm{MM}^{*}, \mathrm{SH}\right)$; School of Geography \& Environmental Studies, Faculty of Science, Engineering \& Technology, University of Tasmania, Hobart 7001, Tasmania, Australia (CS); School of Biological Sciences, Faculty of Science, Engineering \& Technology, University of Tasmania, Hobart 7001, Tasmania, Australia (DB, SW). *Author for correspondence. Email: mike.macphail@anu.edu.au

Cut-back of a sea-cliff at Hannant Inlet in remote South-West Tasmania has exposed Oligocene clays buried under Late Pleistocene "colluvium" from which abundant wood fragments protrude. The two units are separated by a transitional interval defined by mixed Oligocene and Pleistocene microfloras. Microfloras preserved in situ in the clay provide a link between floras in Tasmania and other Southern Hemisphere landmasses following onset of major glaciation in East Antarctica during the Eocene-Oligocene transition (c. $34 \mathrm{Ma}$ ). The Late Pleistocene "colluvium" preserves abundant fossil pollen of the shrub conifer genus Pherosphaera (al. Microstrobos). Assuming the parent plants had the same upper subalpine-alpine ecology as living Pherosphaera hookeriana, the microflora provides evidence for cold, wet conditions in the Port Davey lowlands during a low sea-level stand. The same data highlight the failure of Pherosphaera to regain its Pleistocene distribution during the Postglacial period. Our data are inconclusive whether Late Pleistocene conditions in Hannant Inlet were periglacial, i.e., the Oligocene sediments were turbated by freeze-thaw processes, or have been reworked by fluvial processes into the Pleistocene "colluvium". Nevertheless, the inferred cold-climate is consistent with the former hypothesis. The sequence is sealed under cross-bedded coarse quartzite gravels of presumed Last Glacial Stage age.
\end{abstract}

Key Words: Cenozoic palynostratigraphy, Tasmania, Antarctica, South America, Oligocene, Pleistocene, Lophosoria, Embothrium, Pherosphaera, trans-oceanic migration

\section{INTRODUCTION}

Eroding coastlines have played a decisive role in our understanding of geological time and ancient life - from the world-famous Siccar Point Unconformity on the Berkshire coast (UK), where in 1788 one of the "fathers of geology" James Hutton first demonstrated the immensity of geologic time (Toghill 2000), to Happisburgh on the Norfolk coast, which preserves evidence that palaeohumans had migrated into northwest Europe by c. 840 ka (Parfitt et al. 2010). Evidence of past human occupation is preserved close to sea level around Tasmania, e.g., engravings on rock outcrops at Preminghana (Mt Cameron West) and Sundown Point on the West Coast (Sims 2013), but the majority of coastal exposures archive much earlier geological and biological events. Examples are the "Great Unconformity", which separates folded Silurian-Devonian slates and horizontallybedded Permian mudstones on the east coast of Maria Island (fig. 1.3 in Corbett 2014), Pleistocene fossil logs, some in apparent growth position at Cox Bight on the far South Coast (Bowman et al. 2013), and possible ice-wedge structures at Rocky Cape on the North-West Coast (Colhoun 1977). Such exposures often are difficult to reach, let alone analyse, but continuing marine cut-back of exposed shoreline deposits means that any associated plant macrofossils and microfossils usually are as well preserved as those recovered from unweathered shales and mudstones in drillcore.
In this paper we describe and illustrate microfloras preserved in recently-exposed Late Paleogene sandy clays and Pleistocene "colluvium" in an eroding sea-cliff at Hannant Inlet in Port Davey on the remote South-West Coast of Tasmania (fig. 1). The sandy clays (Unit A) are sequentially overlain by (i) a Pleistocene gravelly clay colluvium (Unit B) from which numerous large horizontally-bedded wood fragments protrude (pls 1, 2) and (ii) apparently crossbedded coarse quartzite gravels of presumed Last Glacial Stage age (Unit C). Palynostratigraphic evidence (this study) shows the two lower units are separated by a turbated interval distinguished only (MM) by a mixed assemblage of microfossils that otherwise are restricted to either Unit A or to Unit B. Some of the microfossils are the first record of the fossil plant genus (morphogenus) or species (morphospecies) in southern Tasmania. The site, which was previously discovered by geologist Grant Dixon and recorded on the Tasmanian Geoconservation Database maintained by the Tasmanian Department of Primary Industries, Parks, Water \& Environment, was sampled in February 2010 by DB, CS and SW. As is typical in remote South-West Tasmania, it can only be reached by an arduous two-day bushwalk or by boat from Melaleuca Inlet c. $10 \mathrm{~km}$ to the east of Port Davey. Hannant Inlet is an integral part of the South-West National Park and World Heritage Area, and the site augments the already impressive geoheritage values of the Area. 


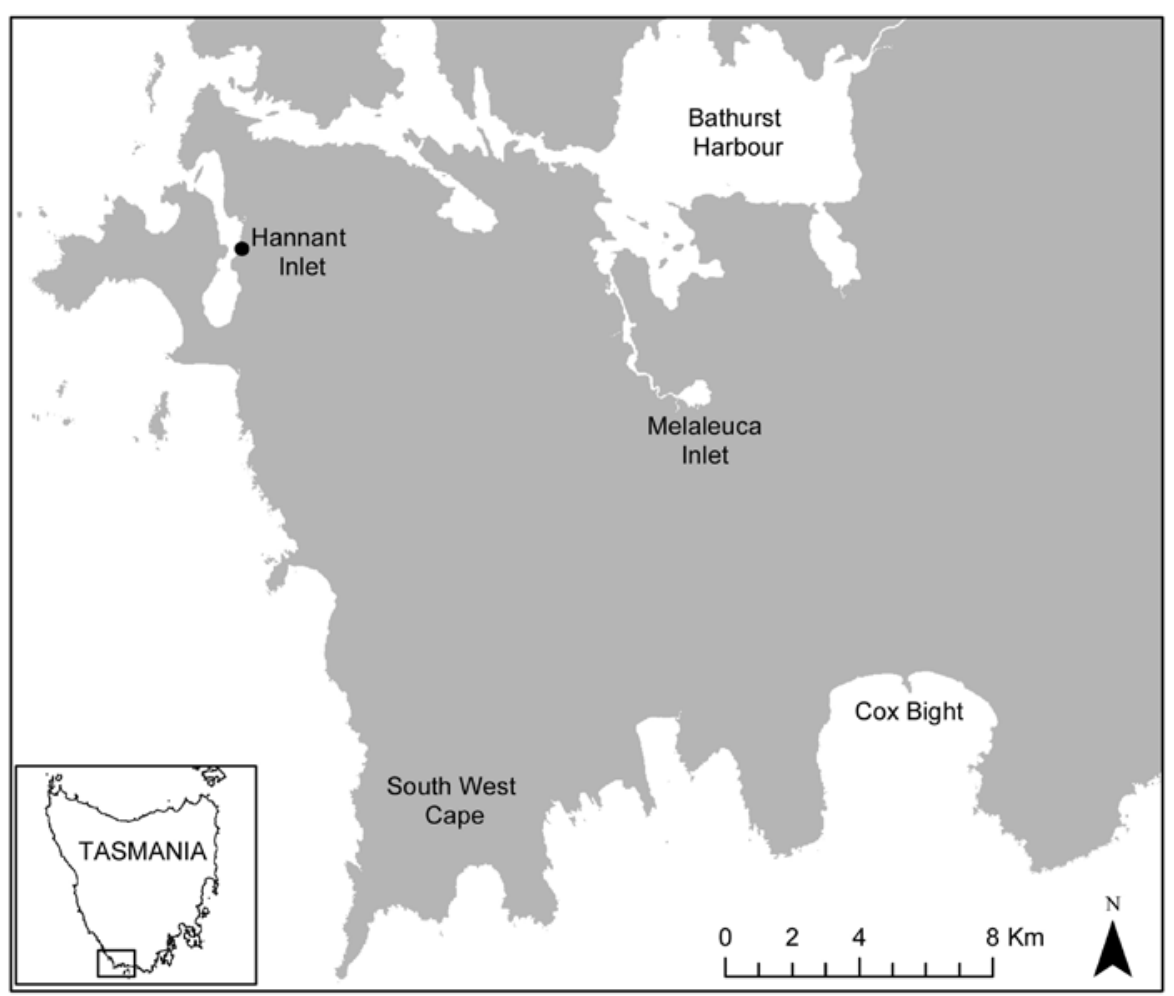

FIG. 1 - Locality map.

\section{PHYSICAL SETTING}

The physical landscape of the Melaleuca-Port Davey region is dominated by extensive lowland buttongrass moorland, and surrounded by the mountains and ranges of the South-West National Park and the expansive interconnected waterways of Port Davey, Bathurst Channel and Bathurst Harbour (Parks and Wildlife Service, Tasmania 2003). Marine fauna in the harbour is unusual in that some taxa are relict, with the closest analogous communities occurring in Milford Sound in New Zealand and in Tierra del Fuego in southern South America. The shallow estuarine complex occupies a drowned river system (ria) incised into deformed, low- to mediumgrade metamorphosed Precambrian rocks, chiefly quartzites, phyllites and schists. The youngest sedimentary rocks in the area are relatively un-metamorphosed conglomerates, sandstones and shales of the Proterozoic Clytie Cove Group (Williams \& Corbett 1977, Williams 1981, 1982).

Higher mountains in the South-West National Park, e.g., Federation Peak (1224 m elevation) and Mt La Perouse (1157 m elevation) were glaciated on several occasions during the Pleistocene and the regional snowline during the Last Glacial Maximum c. $20 \mathrm{ka}$ ago was as low as 600 $\mathrm{m}$ elevation (cf Colhoun et al. 2014). The nearest evidence of Pleistocene glaciation to Hannant Inlet is on Mt Norold (822 m elevation) located c. $25 \mathrm{~m}$ ENE of Hannant Inlet (Stefanski 1957) but there is no evidence that glaciers developed on other ranges in the Melaleuca-Port Davey region or extended onto the coastal lowlands during the Last or earlier Quaternary Glacial Stages. In contrast, it is highly probable that the region experienced severe freezethaw (periglacial) conditions during glacial and stadial episodes (references in Colhoun et al. 2014). During periods of glacio-eustatic low sea levels, the ria becomes a chain of shallow valleys that are connected by deeper channels incised into the bedrock by streams draining mountainous terrain to the north, east and south. At these times, the coastal plain around Port Davey extended seaward of the present shoreline by c. $7-15 \mathrm{~km}$ depending whether the palaeo-coastline is placed at the $100 \mathrm{~m}$ depth- or $150 \mathrm{~m}$ depth-contours, respectively.

The present-day climate is cool and wet (microtherm, perhumid), with evapo-transpiration further reduced by the high incidence of cloud cover. Abundant rainfall and repeated anthropogenic wildfires have allowed buttongrass (Gymnoschoenus sphaerocephalus (R.Br.) Hook.f. moorland to expand from the poorly drained valley flats onto the upper slopes of most ranges; wildfires have restricted woody communities to fire-protected sites such as deeper gullies, southeast facing slopes, islands within Bathurst Harbour, and coastal wetlands surrounding creeks and lagoons (see Wood et al. 2011a, 2011b). These wet communities include: (i) rainforest scrub, mixed-forest and closed rainforest types dominated by Nothofagus cunninghamii (Hook.) Oerst, Phyllocladus aspleniifolius (Labill.) Hook.f., Eucryphia lucida (Labill.) Baill., Atherosperma moschatum Labill. and Anopterus glandulosus Labill., gallery rainforest dominated by Lagarostrobos franklinii (Hook.f.) Quinn, wet sclerophyll scrub dominated by Leptospermum or Melaleuca with a sparse overstorey of Eucalyptus nitida Hook.f. and (ii) Eucalyptus nitida wet sclerophyll forest in which Nematolepis squamea (Labill.) P.G.Wilson and Pittosporum bicolor Hook. are common and where rainforest species, tall sedges (Gahnia grandis (Labill. S.T.Blake)) and ferns such as Pteridium esculentum (G.Forst.) Cockayne also may occur. Lowland herbaceous communities are mostly confined to the coastal plain and include small areas of Chenopodiaceae-dominated saltmarsh within the estuaries. 


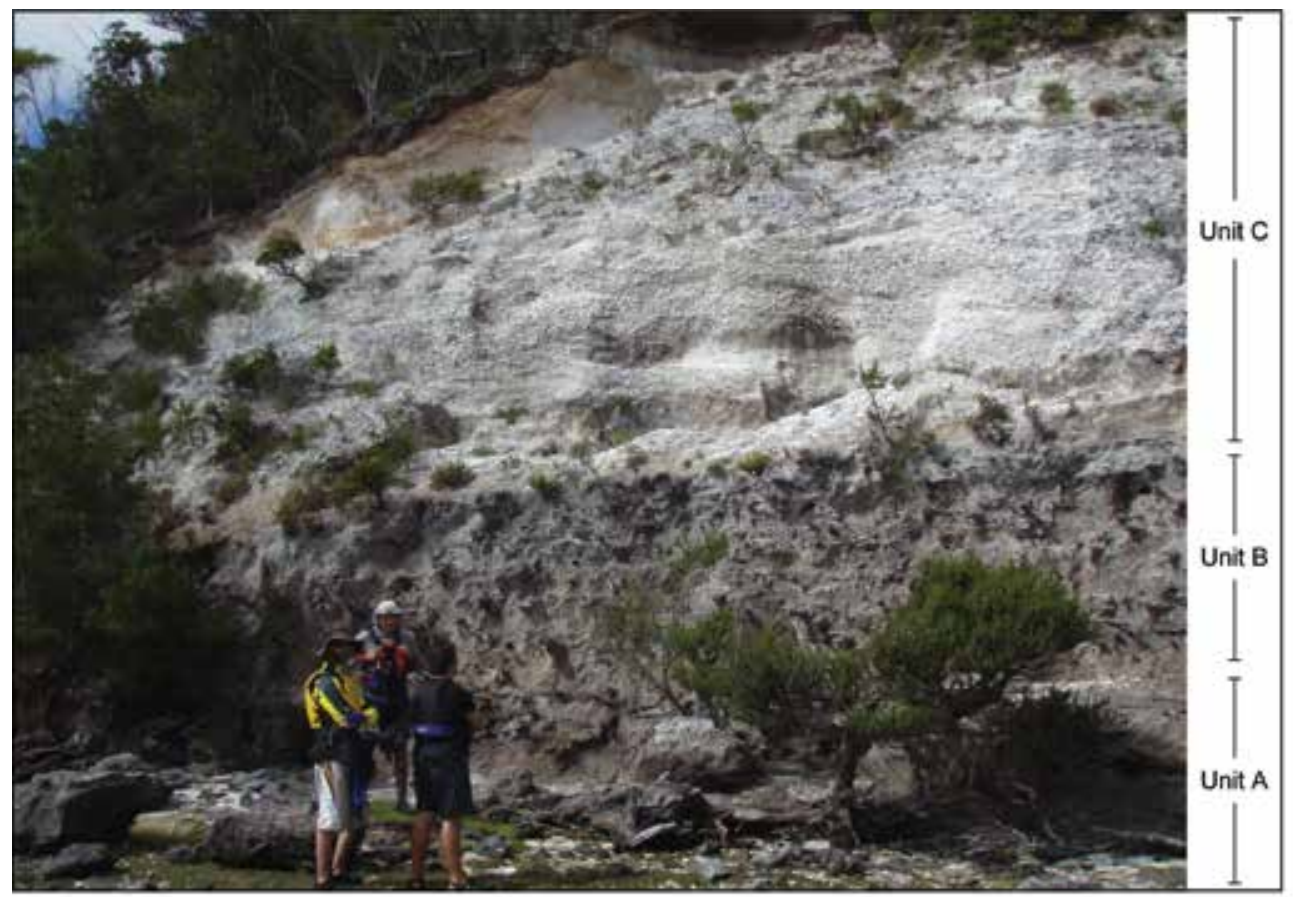

PLATE 1 - View of sea-cliff at Hannants Inlet (photograph by C. Sharples).

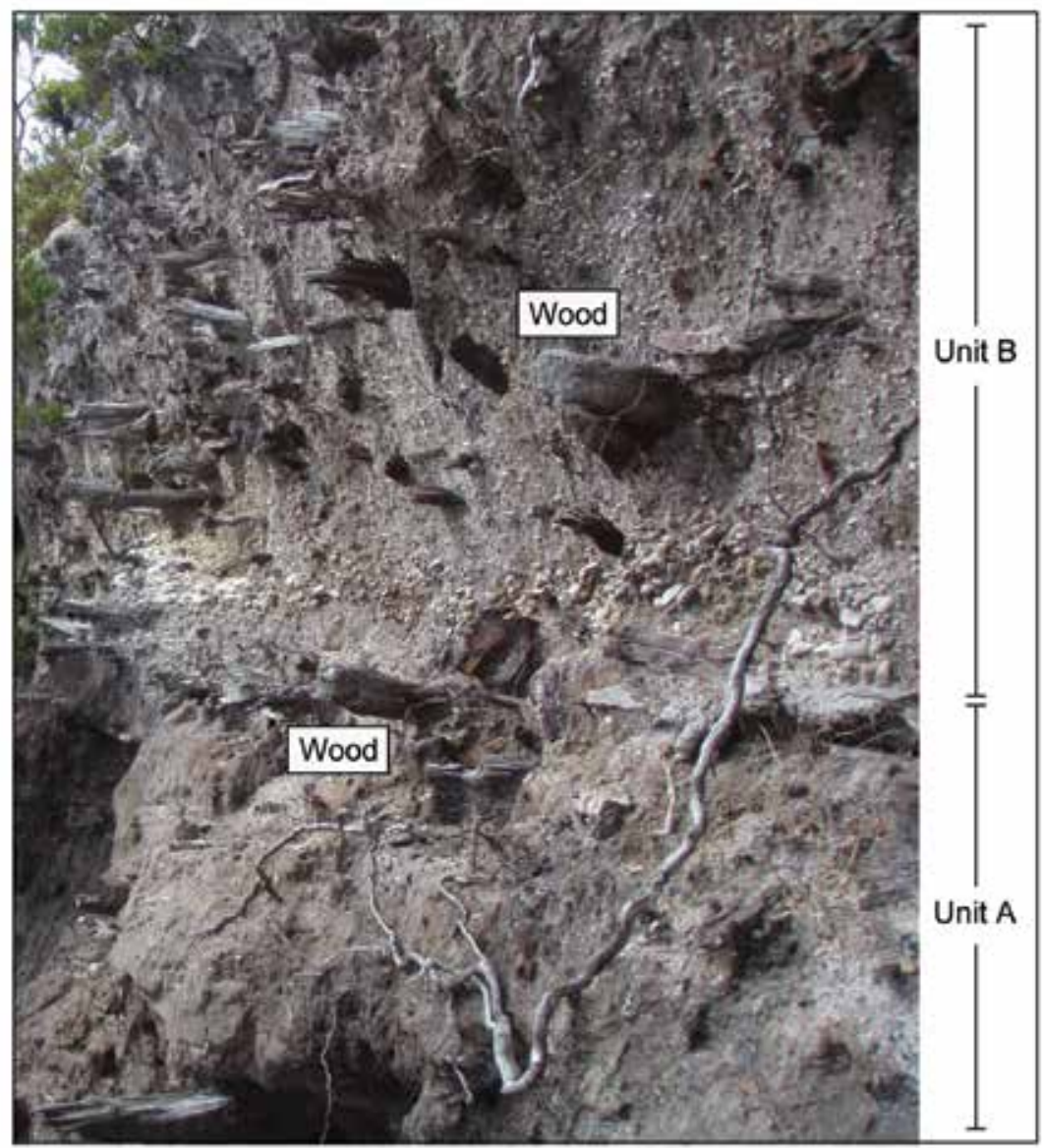

PLATE 2 - Close up view of Hannant Inlet section showing sampled facies (photograph by C. Sharples). 
None of the mountain ranges in the Melaleuca-Port Davey area rise above the climatic limit for tree-growth in southern Tasmania but cold climate floras including Astelia and Microcachrys occur on some of the summit ridges on $\mathrm{Mt}$ Norold, Mt Rugby (734 m elevation) Mt Council (801 $\mathrm{m}$ elevation) and the South-West Cape Range, (684 m elevation), presumably as a result of exposure to extreme winds. Pherosphaera hookeriana W.Archer bis occurs in subalpine-alpine environments at elevations above $1000 \mathrm{~m}$ on Mt Anne and Precipitous Bluff c. $60 \mathrm{~km}$ to the NE and WSW of Hannant Inlet, respectively (fig. 2). Based on data from 26 collection localities, the climate preferences of this shrub conifer include a mean annual temperature of $7.5^{\circ} \mathrm{C}$, with an average minimum in the coldest month of $0.1^{\circ} \mathrm{C}$, and a mean annual precipitation of $1613 \mathrm{~mm}$ (Brown et al. 1983, Biffen et al. 2011).

\section{Hannant Inlet}

The inlet, which occupies a north-south-trending depression eroded into Precambrian metasediments, is bounded to the east by the South-West Cape Range and to the west by a complex peninsula of low hills and bedrock outcrops (maximum elevation $117 \mathrm{~m}$ ) overlain by Quaternary alluvium and colluvium. As with other shallow inlets in Port Davey, the embayment is marine only during periods of high relative sea levels, and the present shoreline is likely to have developed only after sea levels stabilised around their present position some $6 \mathrm{ka}$ ago. For example, the southwest end of the Inlet is separated from the open ocean (Stephens Bay) by tidal flats, mud banks and partly stable transgressive sand dunes. The deposits of "bedded gravels and cobbles" outcropping midway down the eastern shoreline in the inlet and putatively

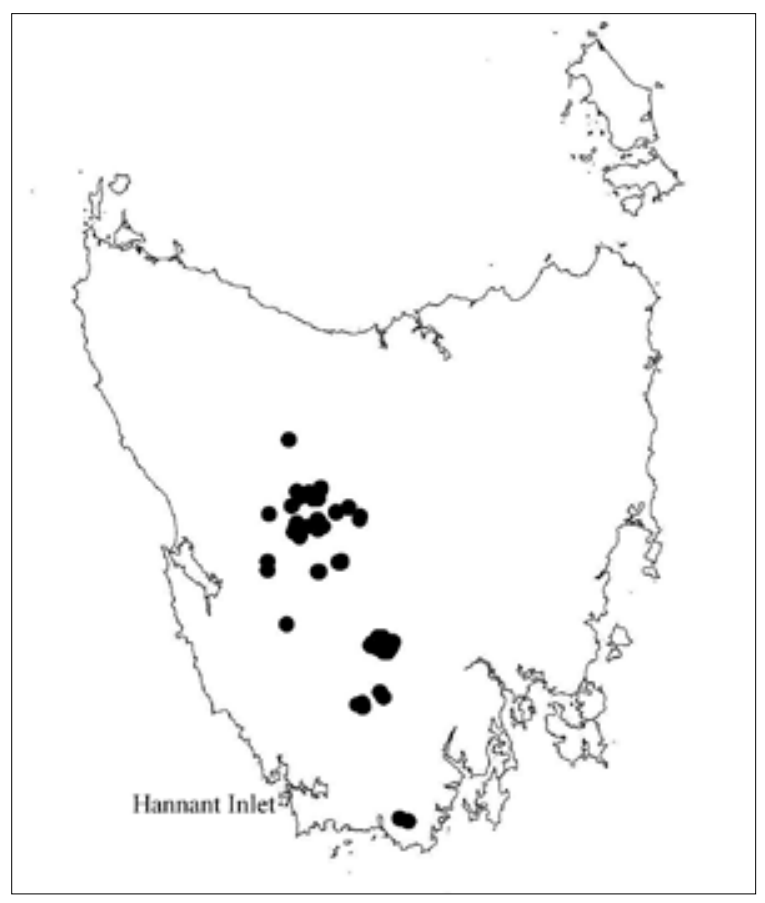

FIG. 2 - Present-day distribution of Pherosphaera hookeriana (after Brown et al. 1983). identified as a raised beach by Williams (1982), is our Unit $\mathrm{C}$ in the Hannant Inlet sea-cliff. Imbricate gravels on the opposite (western) shore of the inlet are different from Unit $\mathrm{C}$ and other putative raised beach deposits mapped in the Port Davey; their presumed Pleistocene age has yet to be confirmed (C. Sharples unpubl. observations). In contrast, palaeochannel sediments and peats at Melaleuca Inlet, which preserve the micro- and macrofossil remains of sclerophyll and rainforest plants, are known to date to the Late Pleistocene and/or Holocene (Jordan \& Hill 1991, Jordan et al. 1991, Thomas 1995, Macphail et al. 1999). Soil around the buried logs at Cox Bight preserves pollen of the cold climate shrub conifers Pherosphaera, Microcachrys, Ericaceae and an Asteraceae (Cassinia arcuata-type) now restricted to mainland Australia (Bowman et al. 2013). As far as we know, sites analogous to the Hannant Inlet outcrop have not been found on the South-West Coast or elsewhere around the Tasmanian coastline.

\section{Site details}

The fossiliferous sediments outcrop near the base of a c. $20 \mathrm{~m}$ high sea-cliff at $43.3694^{\circ} \mathrm{S} 145.9987^{\circ} \mathrm{E}$ (GPS point, GDA94 datum), located approximately midway along the eastern shoreline of the inlet. The section could not be formally measured because of its remote location and time constraints, but cursory field observations (CS) suggest that the lower units had only been recently exposed by wave action. At this time, the visible lithostratigraphy comprised three units (heights measured relative to high water mark are approximate only):

\section{5-15 m AHD (Unit C) coarse cross-bedded quartzite gravels lacking organic remains. \\ 0.5-4.5 m AHD (Unit B) coarsely bedded gravelly clays with horizontally-bedded and aligned wood fragments. \\ 0.0-0.5 m AHD (Unit A): sandy clays lacking obvious plant remains.}

For the purposes of this paper we have adopted the neutral position that Units B and C are "colluvium" although we recognise the sedimentary architecture of these units implies both may be alluvial fan units deposited in relatively high energy fluvial environments. Palynological evidence (this paper) indicates the gravelly clays (Unit B) and underlying sandy clays (Unit A) are separated by a turbated interval that includes a thin layer of water-worn pebbles. Field observations suggest this interval is the basal stratum in Unit $\mathrm{B}$, consistent with palynostratigraphic evidence showing clay overlying the pebble layer preserves a mixed Paleogene/ Pleistocene microflora (table 1). This could be due to fluvial processes reworking palynomorphs from the underlying sandy clays. However, an alternative interpretation is that the interval is a separate unit (solifluction deposit) emplaced under periglacial (freeze-thaw) conditions. Microfossil data indicate that Unit A, initially identified as a palaeosol (Bowman et al. 2013), is more likely to have accumulated in a mire on the eastern side of the Hannant Inlet palaeovalley. 
TABLE 1 - Stratigraphic distribution of plant microfossils at Hannant Inlet and Fossil Bluff, Wynyard

\begin{tabular}{lccccc}
\hline Fossil taxon & $\begin{array}{c}\text { Nearest living relative } \\
\text { [genus/family] }\end{array}$ & \multicolumn{1}{c}{ Hannant Inlet } & Fossil \\
\cline { 3 - 4 } & & 3 & 2 & 1 & Bluff \\
\hline Pollen count & & 531 & 434 & 310 & 800
\end{tabular}

\section{CRYPTOGAMS, GYMNOSPERMS}

Algae, mosses, liverworts

Baculatisporites spp.

Botryococcus

Bryosporis

Stereisporites australis

Ferns \& fern allies

Baculatisporites disconformis

Cyatheacidites annulatus

Cyathidites australis/minor

Cyathidites paleospora

Cyathidites subtilis

Dictyophyllidites arcuatus

Foveotriletes balteus

Foveotriletes lacunosus

Foveotriletes palaequetrus

Herkosporites elliottii

Ischyosporites cf lachlanensis

Gleicheniidites spp.

Kuylisporites waterbolkii

Laevigatosporites ovatus/major

Latrobosporites crassus

Latrobosporites marginis

Matonisporites ornamentalis

Monolites alveolatus

Ophioglossisporites lacunosus

Peromonolites vellosus

Polypodiaceoisporites cf. retirugatus

Polypodiisporites histiopteroides

Polypodiisporites spp.

Rugulatisporites spp.

Trilites tuberculiformis

Verrucosisporites cristatus

Verrucosisporites kopukuensis

\section{Gymnosperms}

Araucariacites australis

Araucariacites cf. australis

Cupressacites

Dacrycarpites australiensis

Dacrydiumites florinii

Dilwynites granulatus

Microalatidites palaeogenicus

Microcachryidites antarcticus

Phyllocladidites mawsonii

Parvisaccites catastus

Podocarpidites spp.

Podosporites sp (bald sacci)

Podosporites erugatus

Podosporites parvus/microsaccatus
Hepaticae

Botryococcus

Musci

Sphagnum (Sphagnaceae)

Hymenophyllaceae

Lophosoria (Lophosoriaceae)

Cyatheaceae

Cyathea (Cyatheaceae)

Cyathea (Cyatheaceae)

Gleicheniaceae

Lycopodium sensu lato (Lycopodiales)

Lycopodium

Lycopodium sensu lato (Lycopodiales)

Lycopodium deuterodensum-type (Lycopodiaceae)

Dicksoniaceae?

Gleicheniaceae

Hemitelia-type (Cyatheaceae)

Blechnaceae, Schizaeaceae

Lycopodiales

Lycopodiella lateralis (Lycopodiaceae)

Dicksonia antarctica (Dicksoniaceae)

Belvisia-type (Polypodiaceae)

Ophioglossum/Phylloglossum

Blechnaceae?

Pteris (Adiantaceae)

Histiopteris incisa (Dennstaedtiaceae)

Davalliaceae/Polypodiaceae

Calochlaena (Thyrsopteridaceae)

Dicksoniaceae

Lygodium (Schizaeaceae)

Lygodium (Schizaeaceae)

Araucaria (Araucariaceae)

Agathis (Araucariaceae)

Cupressaceae/Taxodiaceae

Dacrycarpus (Podocarpaceae)

Dacrydium (Podocarpaceae)

Agathis/Wollemia (Araucariaceae)

Phyllocladus (Podocarpaceae)

Microcachrys (Podocarpaceae)

Lagarostrobos (Podocarpaceae)

Dacrydium bidwillii-type (Podocarpaceae)

Podocarpus/Prumnopitys(Podocarpaceae)

Podocarpus/Prumnopitys (Podocarpaceae)

Pherosphaera/Microstrobos (Podocarpaceae)

cf. Microcachrys (Podocarpaceae)

$\begin{array}{cccc} & 1 \% & 1 \% & \mathrm{x} \\ + & & + & \\ \mathrm{x} & \mathrm{x} & + & \\ & + & 3 \% & \mathrm{x} \\ & & & \\ & \mathrm{x} & \mathrm{x} & 2 \% \\ \mathrm{x} & + & & + \\ & \mathrm{x} & + & \\ & \mathrm{x} & & 10 \% \\ & & \mathrm{cf} . & \mathrm{x} \\ & & & +\end{array}$

$\mathrm{x}$

$6 \%$

30\% $\quad+\quad+$

$+$

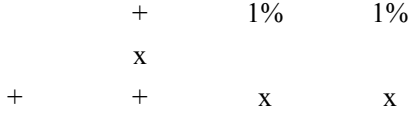

$2 \%$

$\mathrm{X}$

$\mathrm{X}$

$\mathrm{x}$

$\mathrm{x}$

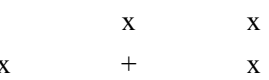

$+\quad 1 \% \quad 8 \%$

$+\quad x+$

X +

$\begin{array}{ccc}5 \% & 6 \% & 5 \% \\ & \mathrm{x} & \end{array}$

$1 \% \quad 2 \% \quad 2 \% \quad 2 \%$

$\begin{array}{cccc}+ & 1 \% & + & \\ 3 \% & 12 \% & 6 \% & 3 \%\end{array}$

$+\quad+\quad+\quad x$

$19 \% \quad 8 \% \quad 7 \%$

$1 \%$

$15 \% \quad 10 \% \quad \mathrm{x}$ 
TABLE 1 (cont.) - Stratigraphic distribution of plant microfossils at Hannant Inlet and Fossil Bluff, Wynyard

\begin{tabular}{|c|c|c|c|c|c|}
\hline \multirow[t]{2}{*}{ Fossil taxon } & \multirow{2}{*}{$\begin{array}{c}\text { Nearest living relative } \\
\text { [genus/family] }\end{array}$} & \multicolumn{3}{|c|}{ Hannant Inlet } & \multirow{2}{*}{$\begin{array}{l}\text { Fossil } \\
\text { Bluff }\end{array}$} \\
\hline & & 3 & 2 & 1 & \\
\hline \multicolumn{6}{|l|}{ ANGIOSPERMS } \\
\hline \multicolumn{6}{|l|}{ Angiosperms (herbaceous taxa) } \\
\hline Aglaoreidia qualumis & Typhaceae? & & & & $\mathrm{x}$ \\
\hline Arecipites spp. & cf. Arecaceae & & & $\mathrm{x}$ & $\mathrm{x}$ \\
\hline Chenopodipollis chenopodiaceoides & Amaranthaceae-Chenopodiaceae & + & & & $\mathrm{x}$ \\
\hline Cyperaceaepollis & Cyperaceae & $1 \%$ & $\mathrm{x}$ & + & + \\
\hline Droseridites sp. & Drosera (Droseraceae) & $\mathrm{x}$ & $\mathrm{x}$ & & \\
\hline Graminidites & Poaceae & $\mathrm{x}$ & & & \\
\hline Liliacidites bainii & Arecaceae/Liliaceae & & & $\mathrm{x}$ & \\
\hline Liliacidites spp. & Liliales & & $1 \%$ & $4 \%$ & $\mathrm{x}$ \\
\hline Luminidites sp. & cf. Dianella (Liliaceae) & & & $\mathrm{x}$ & \\
\hline Milfordia hypolaenoides & Restionaceae & $20 \%$ & + & + & $\mathrm{x}$ \\
\hline Periporopollenites vesicus & Caryophyllaceae? & & & & $\mathrm{x}$ \\
\hline Polycolpites sp. & Wahlenbergia (Campanulaceae) & & & & \\
\hline Striacolporites & Scaevola-type (Goodeniaceae) & & & & \\
\hline Tricolpites stylidioides & Stylidium (Stylidiaceae) & $\mathrm{x}$ & & & \\
\hline Tricolpites sp. A & Brassicaceae & $1 \%$ & & & \\
\hline Tricolporites sp. A & Dichosciadium (Apiaceae) & + & & & \\
\hline Tricolporites sp. B & Apiaceae-type & + & + & & \\
\hline \multicolumn{6}{|c|}{ Angiosperms (woody and unassigned taxa) } \\
\hline Acaciapollenites myriosporites & Acacia (Mimosaceae) & & $\mathrm{x}$ & & \\
\hline Ailanthipites paenestriatus & includes Anacardiaceae & & $\mathrm{x}$ & + & $\mathrm{x}$ \\
\hline Banksieaeidites sp. cf. serrata & Banksia serrata-type (Proteaceae) & & $\mathrm{x}$ & & $\mathrm{x}$ \\
\hline Beaupreaidites elegansiformis & Beauprea (Proteaceae) & & $\mathrm{x}$ & + & $\mathrm{x}$ \\
\hline Beaupreaidites verrucosus & Beauprea (Proteaceae) & & & $\mathrm{x}$ & \\
\hline Bluffopollis scabratus & Strasburgeriaceae & & & $\mathrm{x}$ & \\
\hline Canthiumidites cf. oblatus & Canthium sensu lato (Rubiaceae) & & $\mathrm{x}$ & cf. & \\
\hline Clavatipollenites glarius & Ascarina (Chloranthaceae) & & & cf. & + \\
\hline Concolpites leptos & Gillbeea-type (Cunoniaceae) & & & & $\mathrm{x}$ \\
\hline Crotonipollis sp. & Ricinocarpos (Euphorbiaceae) & + & & & \\
\hline Cupanieidites orthoteichus & Sapindaceae tribe Cupanieae & & & + & \\
\hline Granodiporites nebulosus & Embothrium (Proteaceae) & & $\mathrm{x}$ & $?$ & $\mathrm{x}$ \\
\hline Gyropollis psilatispora & Gyrostemonaceae & & & $\mathrm{x}$ & \\
\hline Ericipites sp A & Sprengelia-type (Epacridaceae) & $1 \%$ & $1 \%$ & $\mathrm{x}$ & \\
\hline Ericipites spp. & Epacridaceae-Ericaceae & $3 \%$ & $3 \%$ & $2 \%$ & + \\
\hline Gyropollis psilatus & Gyrostemonaceae & & & & \\
\hline Haloragacidites harrisii & Casuarinaceae & $19 \%$ & $10 \%$ & $11 \%$ & $6 \%$ \\
\hline Ilexpollenites anguloclavatus & Ilex (Aquilifoliaceae) & & & & $\mathrm{x}$ \\
\hline Monotocidites galeatus & Monotoca (Euphorbiaceae) & + & $1 \%$ & + & \\
\hline Monotocidites sp. & Leucopogon (Euphorbiaceae) & & & & \\
\hline Myrtaceidites eucalyptoides & Eucalyptus sensu lato (Myrtaceae) & + & $1 \%$ & & $\mathrm{x}$ \\
\hline Myrtaceidites leptospermoides & Leptospermum-type (Myrtaceae) & + & & cf. & \\
\hline Myrtaceidites $\mathrm{cf}$. lipsis & Eucalyptus (Myrtaceae) & + & & & \\
\hline Myrtaceidites parvus-mesonesus & Myrtaceae & & $2 \%$ & + & $\mathrm{x}$ \\
\hline Myrtaceidites verrucosus & Austromyrtus-type (Myrtaceae) & & $\mathrm{x}$ & & $\mathrm{x}$ \\
\hline Myrtaceidites sp. A & Melaleuca-type (Myrtaceae) & & $\mathrm{x}$ & & \\
\hline Nothofagidites asperus & Nothofagus(Lophozonia) spp. e.g. N. cunninghamii & $1 \%$ & $\mathrm{x}$ & $3 \%$ & $4 \%$ \\
\hline Nothofagidites brachyspinulosus & Nothofagidites (Fuscospora) spp. e.g. N. gunnii & + & $1 \%$ & $3 \%$ & $2 \%$ \\
\hline Nothofagidites emarcidus-heterus & Nothofagus(Brassospora) spp. (Nothofagaceae) & & $15 \%$ & $18 \%$ & $34 \%$ \\
\hline Nothofagidites falcatus & Nothofagus(Brassospora) spp. (Nothofagaceae) & & $1 \%$ & $2 \%$ & + \\
\hline Nothofagidites flemingii & Nothofagus(Brassospora) spp. (Nothofagaceae) & & + & $\mathrm{x}$ & $\mathrm{x}$ \\
\hline Nothofagidites vansteenisii & Nothofagus(Brassospora) spp. (Nothofagaceae) & & & $\mathrm{cf}$. & $\mathrm{x}$ \\
\hline Nuxpollenites sp. & Dodonaea-triquetra-type (Sapindaceae) & & $\mathrm{x}$ & & $\mathrm{x}$ \\
\hline
\end{tabular}


TABLE 1 (cont.) - Stratigraphic distribution of plant microfossils at Hannant Inlet and Fossil Bluff, Wynyard

\begin{tabular}{|c|c|c|c|c|c|}
\hline \multirow[t]{2}{*}{ Fossil taxon } & \multirow{2}{*}{$\begin{array}{c}\text { Nearest living relative } \\
\text { [genus/family] }\end{array}$} & \multicolumn{3}{|c|}{ Hannant Inlet } & \multirow{2}{*}{$\begin{array}{l}\text { Fossi } \\
\text { Bluff }\end{array}$} \\
\hline & & 3 & 2 & 1 & \\
\hline Palaeocoprosmadites zelandiae & Coprosma-type (Rubiaceae) & $\mathrm{x}$ & & & \\
\hline Paripollis sp. & Epacris (Epacridaceae) & $\mathrm{x}$ & & & \\
\hline Poluspissusites ramosus & Goodeniaceae & $\mathrm{x}$ & $\mathrm{x}$ & & \\
\hline Polycolpites sp. sp. & unknown & & $1 \%$ & $\mathrm{x}$ & \\
\hline Propylipollis annularis & Xylomelum (Proteaceae) & & $\mathrm{x}$ & $\mathrm{x}$ & $\mathrm{x}$ \\
\hline Proteacidites obscurus & Agastachys (Proteaceae) & & $1 \%$ & $3 \%$ & $\mathrm{x}$ \\
\hline Proteacidites persoonioides $\mathrm{ms}$ & Persoonia (Proteaceae) & + & & & \\
\hline Proteacidites scaboratus & Proteaceae & & $\mathrm{x}$ & $\mathrm{x}$ & \\
\hline Proteacidites stipplatus & Proteaceae & & $1 \%$ & $\mathrm{x}$ & \\
\hline Proteacidites tuberculatus & Proteaceae & & & & $\mathrm{x}$ \\
\hline Proteacidites spp. & Proteaceae & & $2 \%$ & $2 \%$ & $\mathrm{x}$ \\
\hline Pseudowinterapollis cranwellae & Tasmannia (Winteraceae) & + & $\mathrm{x}$ & & $\mathrm{x}$ \\
\hline Psiladiporites sp. & Alyxia (Apocynaceae) & & & & $\mathrm{x}$ \\
\hline Quintiapollis psilatispora & Quintinia (Grossulariaceae) & & & $\mathrm{x}$ & $\mathrm{x}$ \\
\hline Rhoipites ampereaformis & Amperea (Euphorbiaceae) & + & $\mathrm{x}$ & & $\mathrm{x}$ \\
\hline Rhoipites sp. & Apiaceae & & & & \\
\hline Rhoipites spp. & includes Rutaceae & & + & + & $\mathrm{x}$ \\
\hline Rubipollis oblatus & Canthium-type & $\mathrm{x}$ & & & $\mathrm{x}$ \\
\hline Sapotaceoidaepollenites rotundus & Sapotaceae & & & $\mathrm{x}$ & \\
\hline Spinitricolporites & unknown angiosperm & & & $\mathrm{x}$ & \\
\hline Stephanocolpites oblatus & Haloragodendron/Glischrocaryon (Haloragaceae) & & $\mathrm{x}$ & & \\
\hline Tetracolporites palynius & cf. Alectryon & & cf. & $\mathrm{x}$ & \\
\hline Tricolpites sp. B & Hibbertia-type (Dilleniaceae) & $\mathrm{x}$ & & & \\
\hline Tricolpites spp. & numerous families & & $2 \%$ & $3 \%$ & \\
\hline Tricolporites leuros & unknown & & & & $\mathrm{x}$ \\
\hline Tricolporites sp. C & Anopterus/Donatia (Escalloniaceae/Donatiaceae) & & & $\mathrm{x}$ & \\
\hline Tricolporites sp. D & Pomaderris apetala (Rhamnaceae) & + & & & \\
\hline Tricolporites spp. & numerous families & & + & + & + \\
\hline Tricolporopollenites endobalteus & Mallotus-type (Euphorbiaceae) & & & $\mathrm{x}$ & \\
\hline Triporopollenites ambiguus & Telopea (Proteaceae) & & & & $\mathrm{x}$ \\
\hline Tubulifloridites antipoda & Tubuliflorae (Asteraceae) & $1 \%$ & & & \\
\hline Tubulifloridites pleistocenicus & Cassinia arcuata-type (Asteraceae) & + & & & \\
\hline Tubulifloridites simplis & Tubuliflorae (Asteraceae) & $1 \%$ & & & \\
\hline
\end{tabular}

\section{PALYNOSTRATIGRAPHY}

One sample collected from each of the two main sedimentary units and one from the "turbated" interval were taken for palynostratigraphic analysis, i.e., from within Unit A (Sample 1 ), from the "turbated" interval near the apparent base of Unit B (Sample 2), and from the clay matrix surrounding a piece of wood mid-section within Unit B (Sample 3). Pieces of wood from Unit B were collected for AMS radiocarbon dating and to determine whether the source could be identified. Results of the latter were inconclusive although a gymnosperm origin seems likely.

Trial processing (SH) of samples sent to the Australian National University revealed the basal sandy clay (unit A) was considerably older than the Holocene to Last Interglacial age predicted for the sequence at the time of sampling. Additional sub-samples (MM) were reprocessed for pollen, spores (miospores) and other plant microfossils by Core Laboratories (Australia) using standard techniques of oxidation and microfiltration (see Traverse 1988). Palynostratigraphic criteria used to infer the maximum, preferred and minimum age limits of Units A and B are based on morphospecies time-distributions in the offshore Gippsland and Bass Basins in Bass Strait, Victoria (Partridge 1999, 2006, A.D. Partridge \& M.K. Macphail unpubl. data). Geological ages (Ma) are in accordance with the revised timescale of Ogg et al. (2008). Where possible, fossil species are cited under their fossil (morphospecies) names, with the nearest known living relatives (NLRs) given in parentheses. A selection of age-diagnostic, phytogeographically significant, and previously unrecorded morphospecies are illustrated in pls 3-5. 


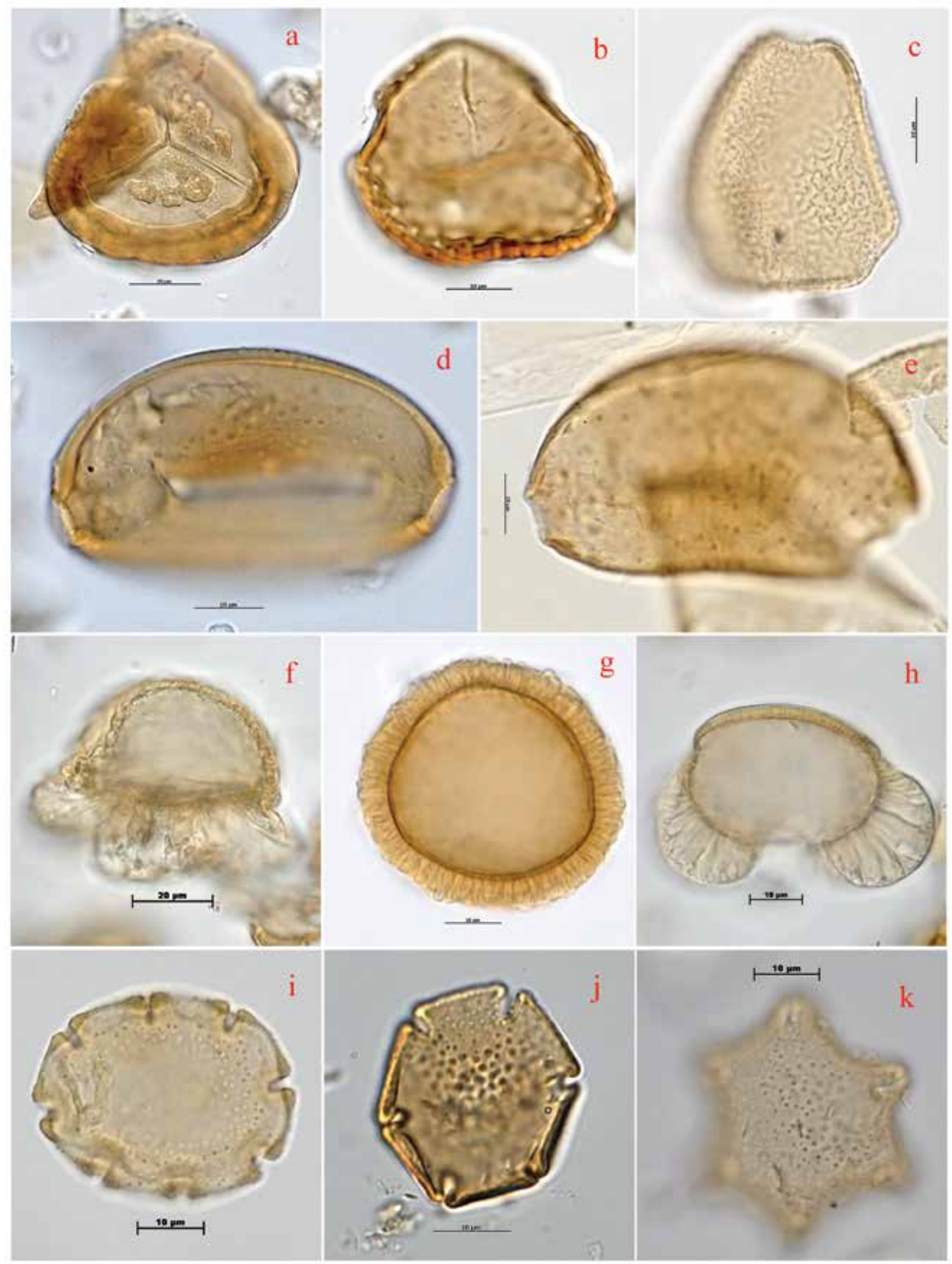

PLATE 3 - Age-diagnostic morphospecies preserved in Unit A (Sample 1) a. Cyatheacidites annulatus (Lophosoria); b. Foveotriletes palaequetrus (Lycopodium); c. Proteacidites stipplatus (extinct Proteaceae clade); d \& e. Granodiporites nebulosus (Embothrium); f. Dacrycarpites australiensis (Dacrycarpus); g. Dacrydiumites florinii (Dacrydium): h. Parvisaccites catastus (Lagarostrobos bidwilliil biforme); i. Nothofagidites flemingii [Nothofagus (Nothofagus) spp.]; j. Nothofagidites emarcidus [Nothofagus (Brassospora) spp.]; k. Nothofagidites falcatus [Nothofagus (Fuscospora) spp.] 


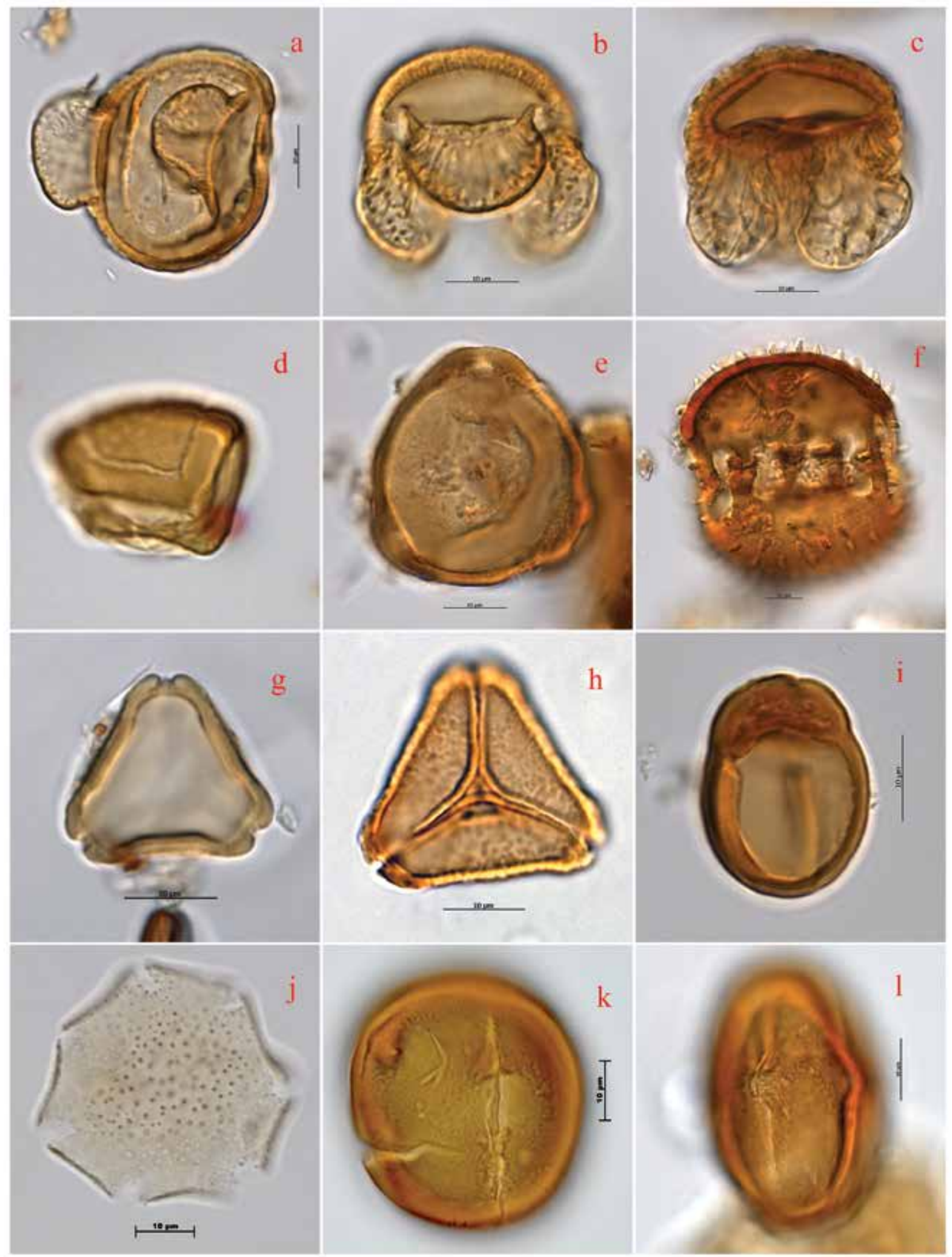

PLATE 4 - Morphospecies recorded in Unit B (Sample 3). Equivalent fossil morphospecies in parentheses. a. Lagarostrobos franklinii (Phyllocladidites mawsonii); b. Pherosphaera hookeriana (Podosporites erugatus); c. Microcachrys tetragona (Microcachryidites antarcticus); d. Acacia monad (Acaciapollenites myriosporites); e. Allocasuarina/Casuarina (Haloragacidites harrisii); f. Drosera (Droseridites sp.); g. Eucalyptus (Myrtaceidites eucalyptoides); h. Melaleuca squamea-type (Myrtaceidites cf. verrucosus); i. Monotoca (Monotocidites galeatus); j. Nothofagus cunninghamii (Nothofagidites asperus); k. cf. Donatia (Tricolporites sp.); I. cf. Anopterus (Tricolporites sp.) 

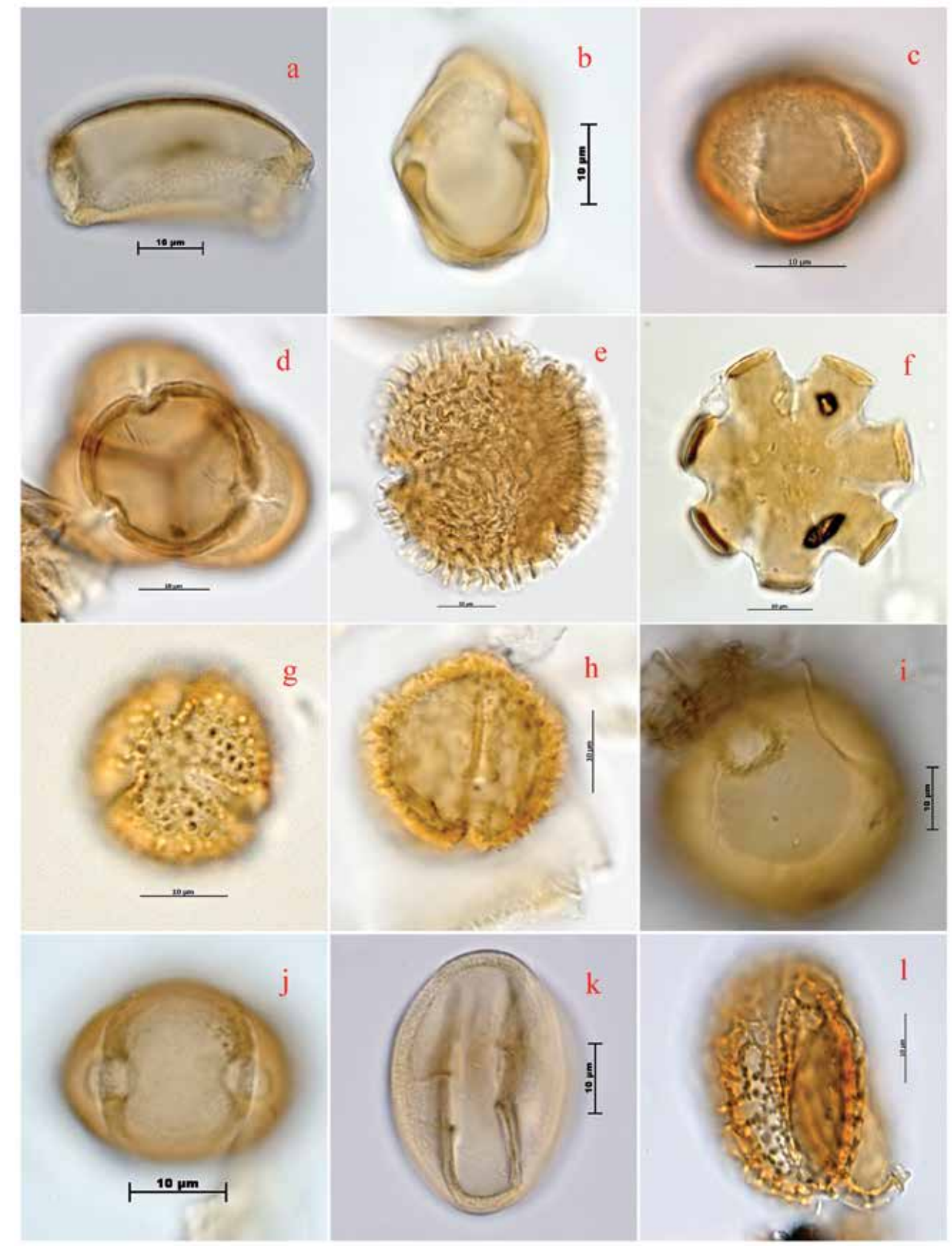

PLATE 5 - Other distinctive pollen morphotypes recorded in Units A \& B. Equivalent fossil morphospecies in parentheses. a. Banksia sp. [Banksieaeidites cf. Banksia serrata (Unit B Sample 2)]. b. Dodonaea triquetra-type [Nuxpollenites sp. (Unit A)]; c. Haloragodendron-type [Stephanocolpites oblatus (Unit B Sample 2)]; d. Ericaceae [Ericipites sp. (Unit A)]; e. Rubiaceae [Canthiumidites sp. (Unit A)]; f. Unidentified taxon [Polycolpites sp. (Unit A)] g-h. Unidentified taxon [Spinitricolporites (al. Tensucolpites) sp. (Unit A)]; i. Unidentified taxon [Triorites cf. orbiculatus (Unit A)]; j. Unidentified taxon cf. Dysoxylum [Tetracolporites sp. (Unit A)]; k. Unidentified taxon [cf. Poluspissusites sp. (Unit A)]; I. Unidentified taxon [Rhoipites sp. (Unit B Sample 2)] 


\section{AMS radiocarbon age control}

Fossil wood from Unit B was submitted for AMS-dating (table 2) but proved to be older than the background radiocarbon limit of c. $45 \mathrm{ka}$. This confirms the colluvium predates the Last Glacial Maximum at c. 22 ka but by how much geologic time is unknown.

\section{Palynostratigraphic age control}

The stratigraphic distribution and estimated relative abundance of all formally-described fossil spores and pollen (miospores) in Samples 1-3 are given in table 1. For comparison purposes, this table includes palynostratigraphic data from an independently dated earliest Miocene deposit that, like the Hannant Inlet site, was located relatively close to palaeocoastline at Fossil Bluff, Wynyard on the North-West Coast of Tasmania (Macphail 1996). Age limits inferred in this paper necessarily assume that morphospecies time-distributions in southernmost Tasmania during the Paleogene-Neogene are comparable with time distributions in the Gippsland and Bass Basins in Bass Strait, and for this reason are expressed as Zone Equivalents (cf. Macphail 1999, Macphail et al. 2014).

\section{Basal sandy clay (Unit A)}

The age limits of Sample 1 collected from within the basal clay unit is Early to Late Oligocene Proteacidites tuberculatus Zone Equivalent, based on (i) Cyatheacidites annulatus (NLR Lophosoria), a spore whose first appearance datum [FAD] defines the lower boundary of the Early Oligocene to late Early Miocene Proteacidites tuberculatus Zone and (ii) Proteacidites stipplatus, a distinctive morphospecies which last appears [LAD] in the Late Oligocene Ophioglossisporites lacunosus Subzone of the Proteacidites tuberculatus Zone (pl. 3a-e). The assemblage includes Granodiporites nebulosus (NLR Embothrium), a morphospecies that last appears in the Early Oligocene Granodiporites nebulosus Subzone of the Proteacidites tuberculatus Zone in the Gippsland Basin but which is found in earliest Miocene sediments at Fossil Bluff (cf. Quilty 1972, Macphail 1996). Other species which first or last appear in the Proteacidites tuberculatus Zone in southeast Australia include Nuxpollenites sp. (Dodonaea triquetra-type) and Foveosporites palaequetrus (Lycopodium), and Aglaoreidia qualumis (Typhaceae?), respectively. Several of the more frequently recorded Nothofagus and Podocarpaceae morphospecies potentially were canopy dominants in the Oligocene vegetation (pl. $3 \mathrm{f}-\mathrm{k}$ ).

\section{Gravelly clay "colluvium" (Unit B)}

The stratigraphically lower of the two samples (Sample 2) representing this unit was collected from within the "turbated" interval at the base of Unit B. The "mixed age" microflora included many of the age-diagnostic morphospecies present in Unit A, e.g., Granodiporites nebulosus and Proteacidites stipplatus, as well as morphospecies that first appear in the Late Miocene Foraminisporis bifurcatus Zone in the Gippsland Basin, e.g., Monotocidites galeatus (Monotoca). The former morphospecies have been reworked or turbated from Unit A facies, either at the site or from upslope if Unit A extends inland under the "colluvial" deposits. The minimum age is uncertain but will pre-date the Last Glacial Maximum at c. $22 \mathrm{ka}$ given that the NLRs of one morphotype present in the Sample 2, Nuxpollenites sp., are extinct in Tasmania (cf. Macphail 1996).

The higher of the two samples (Sample 3) was taken from the clay matrix surrounding a piece of fossil wood mid-section within the unit. This clay yielded a typical Late Quaternary cool-cold climate microflora (cf. Colhoun \& van der Geer 1988, 1998), co-dominated by ferns and herbs, in particular Gleicheniaceae (fossil equivalent Gleicheniidites) and Centrolepidaceae-Restionaceae (Milfordia hypolaenoides), but also includes frequentto-common Pherosphaera hookeriana-type (Podosporites erugatus) and Lagarostrobos franklinii (Phyllocladidites mawsonii) pollen. Although these taxa and many other morphospecies in the sample have Paleogene FADs, at least one taxon, Tubulifloridites pleistocenicus (Cassinia arcuata-type), first becomes consistently recorded in the Early Quaternary in southeastern Australia. Two rare taxa in the microflora, Leucopogon (Epacridaceae) and Pomaderris apetala-type (Rhamnaceae) have not been recorded in sediments older than Middle-Late Pleistocene in Tasmania, whilst the absence of Symplocos (Symplocoipollenites austellus) is negative evidence that the sample is younger than Middle Pleistocene (cf. Macphail et al. 1993b, Augustinus \& Macphail 1997). For these reasons and radiocarbon dates exceeding $45 \mathrm{ka}$ (table 1), we infer the mid-upper section

\begin{tabular}{|c|c|c|c|c|c|}
\hline \multirow{2}{*}{ Lab. code } & \multirow{2}{*}{ Sample ID } & \multirow{2}{*}{$\delta^{13} \mathrm{C} \% 0$} & \multicolumn{2}{|c|}{$\rho \mathrm{MC}^{1}$} & \multirow{2}{*}{$\begin{array}{c}{ }^{14} \mathrm{C} \text { ages }(\mathrm{BP}) \\
\text { Mean }\end{array}$} \\
\hline & & & Mean & $1 \delta$ & \\
\hline OZN1072 & Hannant Inlet Sample 1 & -20.4 & 0.00 & 0.03 & $\mathrm{NDFB}^{3}$ \\
\hline OZN1082 & Hannant Inlet Sample 1 & -20.6 & 0.00 & 0.03 & $\mathrm{NDFB}^{3}$ \\
\hline
\end{tabular}


of Unit B is early Late Pleistocene in age. It is possible that the unit is a correlative of the Cox Bight sub-fossil wood deposit, which overlies a poorly sorted sub-round quartzite cobble and gravel deposit (cf. Bowman et al. 2013).

\section{Quartzite gravels (Unit C).}

This $>10$ m thick unit of "clean" angular quartzite fragments lacked visible organic inclusions and was not sampled for this study. The lithology is inconsistent with Unit C being a raised beach deposit (cf. Williams 1982) and, although typed as "colluvium" (this study), the unit, like Unit B, may be alluvium deposited in a high-energy fluvial environment. Whether the clasts are the result of frost-shattering on a quartzite outcrop upslope during the Last Glacial Maximum is uncertain (cf. Colhoun 1977) although both inferences seem likely.

\section{Depositional environments}

Colonies of the green alga Botryococcus and Stereisporites (Sphagnum) spores indicate Unit A accumulated in a fen or cut-off channel on the eastern side of the Hannant Inlet palaeovalley during the Oligocene. High relative pollen abundances of the extinct shrub conifer Podosporites microsaccatus are consistent with this depositional environment (cf. Tulip et al. 1982). In contrast, Unit B was deposited within a Gleicheniaceae-Restionaceae (or Centrolepidaceae) mire fringed by Pherosphera hookeriana and Casuarinaceae, or the sample (Sample 3) incorporates sediment that had accumulated in such an environment during the Late Pleistocene. Gleichenia-Restionaceaedominated "bog" communities are widespread across a range of elevations in South-West Tasmania and one Casuarinaceae species in Tasmania, Allocasuarina monilifera (L.A.S. Johnson) L.A.S. Johnson, extends from near sea-level up to c. $1100 \mathrm{~m}$ elevation. However, few, if any, modern cold-climate mires in Tasmania are surrounded by both Allocasuarina/Casuarina and Pherosphaera hookeriana. The combined data point to Unit B being deposited during a cold interval in the Last Glacial Stage than an earlier glaciation.

\section{Relationship to past sea levels}

Agreement exists that global sea levels were significantly below the present-day level during the earliest Oligocene and significantly higher than at present during the Early Miocene (see Haq et al. 1987, Miller et al. 1996, Houben et al. 2012, Lewis \& Quilty 2009, Quilty 2014) although actual elevations are difficult to estimate for a given location. For example, Quilty \& Seymour (2010) note there is a difference of about $1 \mathrm{~km}$ between Early Miocene shallow marine sediments at $160 \mathrm{~m}$ elevation inland of Temma on the North-West Coast and correlatives at $800 \mathrm{~m}$ water depth south of Tasmania and suggest this involves tilting of the island as well as changes in relative sea level. The same caveat applies to the Late Cenozoic where glacio-eustatic-forced changes in relative sea level are blurred by uplift and tilting (Houshold et al. 2006, Colhoun et al. 2014). All that can be inferred with moderate confidence is that (a) the absence of marine dinoflagellates and other saline indicators imply Unit A was deposited in a non-marine environment, even if Hannant Inlet was open to the Southern Ocean at this time, and (b) cold conditions during the deposition of Unit $B$ is circumstantial evidence that site could have been up to $100-150 \mathrm{~m}$ above sea level, and $7-15 \mathrm{~km}$ inland from the palaeo-coastline during a cold stage within the last or a preceding Pleistocene glaciation (Colhoun et al. 2014). The same constraints apply to the sequence incorporating the sub-fossil logs at Cox Bight.

\section{Palaeovegetation and climate}

All fossil pollen and spore assemblages are a partial record of past floras, with some indication of the relative abundance of the parent plants in the source vegetation. The latter values are strongly biased by the intrinsic pollen production and dispersal characteristics of the plants (Representivity) as well as by depositional environment, which influences the extent to which miospores derived from the regional vegetation are "diluted" by the influx of miospores from plants growing on, around or (fluvio-deltaic contexts) upstream of a site (see Macphail 2007).

These caveats aside, significant to high relative abundances of Araucariaceae, Podocarpaceae, Nothofagus and Casuarinaceae (Gymnostoma?) pollen confirm the dryland vegetation growing on the eastern side of Hannant Inlet during the Oligocene almost certainly was one of numerous variants of the Nothofagus-gymnosperm evergreen temperate rainforest established across Tasmania and southern Australia in general throughout the Late Paleogene (see Macphail et al. 1994, 2014). No modern analogues of this warm temperate rainforest community exist, either because the taxa are extinct in Australia, or because their NLRs have contracted to a small part of their Cenozoic geographic range. For example (NLRs in parentheses), at least six woody taxa are restricted to humid, mesotherm to megatherm climates, viz. Beaupreaidites elegansiformis and B. verrucosus (Beauprea), Bluffopollis scabratus (Strasburgeriaceae), Cupanieidites orthoteichus (Cupanieae), the complex of Nothofagidites emarcidus morphotypes [Nothofagus (Brassospora) spp.], Quintiniapollis psilatispora (Quintinia) and Sapotaceoidaepollenites rotundus (Sapotaceae). In contrast, the taxa likely to have formed a gymnosperm overstorey mostly have NLRs that are confined to cooler (lower mesotherm-microtherm) environments. Examples are Dacrycarpites australiensis (Dacrycarpus), Dacrydiumites florinii (Dacrydium), Microalatidites palaeogenicus (Phyllocladus), Microcachryidites antarcticus (Microcachrys) and Phyllocladidites mawsonii (Lagarostrobos). Several of these taxa were extirpated from Australia as recently as the Late Pliocene (Nothofagus (Brassospora) spp.) or Pleistocene (Dacrycarpus, Dacrydium). Three other clades now are endemic to South America, viz. Cyatheacidites annulatus (Lophosoria), Granodiporites nebulosus (Embothrium) and Nothofagidites flemingii [Nothofagus (Nothofagus) spp.]. The Asteraceae species represented by Tubulifloridites pleistocenicus is likely to have been extirpated from Tasmania by the early Holocene. One probable shrub gymnosperm, 
Podosporites microsaccatus (Microcachrys?), is wholly extinct. Several of the pollen morphotypes have not been recorded previously and/or have no known NLR (pl. 5).

Based on the present-day ecological amplitude of Nothofagus (Brassospora) spp. in New Caledonia and New Guinea (Table 7.4 in Read \& Hope 1996), it is probable that precipitation was more than $2000 \mathrm{~mm}$ pa, and the climate lacked a dry season. For the same reason, mean annual temperatures are estimated to have been at the lower end of the $12-24^{\circ} \mathrm{C}$ (mesotherm) range. Differences in the composition of the rainforest at Hannant Inlet and Fossil Bluff are minor (table 1). For example, only five morphospecies whose Australian NLRs are considered typical of warm to hot (mesotherm-megatherm) environments occur at Fossil Bluff but not at Hannant Inlet, viz. Clavatipollenites glarius (Ascarina), Ilexpollenites anguloclavatus (Ilex), Myrtaceidites verrucosus (Austromyrtustype), Psiladiporites (Alyxia) and Tricolporopollenites endobalteus (Macaranga-Mallotus). Accordingly, the data imply climatic gradients within Tasmania were subdued relative to the present, but, the variations that are observed can equally well be explained by the northward drift of the Australian continent from c. $63^{\circ} \mathrm{S}-58^{\circ} \mathrm{S}$ during the Oligocene to Early Miocene (see Veevers et al. 1991).

The microflora recovered from the "turbated" zone at the base of Unit B (Sample 2) is unreliable evidence for the nature of the Late Pleistocene vegetation although the data confirm that Eucalyptus spp. were very rare and Pherosphaera relatively common in the local vegetation during the period of deposition (cf. Macphail 1979). Reconstruction of the community recorded in Sample 3 is less complicated since the parent plants either grow in the Port Davey area or conceivably could have done so in the geologically recent past. For example, the association of Pherosphaera, Gleicheniaceae, Restionaceae, Epacridaceae, Microcachrys and Apiaceae is typical of bog and herbfield communities in the upper subalpine-alpine zone on many higher mountains in South-West Tasmania. Moderately high relative abundances of Pherosphaera pollen (15\%) are evidence either Pherosphaera hookeriana or a close relative of this cold climate shrub conifer was growing on the site, now some $1000 \mathrm{~m}$ below its present-day lower altitudinal limit on Mt Field (see Macphail 1979). Sclerophyll shrubs or small trees including Allocasuarina/Casuarina are likely to have occupied much the same freely-draining sites as at present although the shrub flora included genera that are unlikely to be found in coastal buttongrass moorland at present, e.g., Ricinocarpos and a pollen morphotype (pl. 5a) that resembles pollen of Banksia serrata L.f., now confined in Tasmania to relict stands around Sisters Beach on the NorthWest Coast and Flinders Island. Assuming Pherosphaera, Apiaceae and Nothofagus (Fuscospora) pollen represent Pherosphaera hookeriana, Dichosciadium ranunculaceum (F.Muell.) Domin and Nothofagus gunnii (Hook.f.) Oerst respectively, mean temperatures were c. $4^{\circ} \mathrm{C}$ and potentially up to $7-8^{\circ} \mathrm{C}$ below present (cf. Colhoun $\&$ van der Geer 1987, 1998, Colhoun et al. 2014).

We emphasise the Late Pleistocene parent plants may include now-extinct clades and that some Tasmanian cold- climate taxa occurred well beyond their modern climatic range in Australia and elsewhere in the Pleistocene. This concept is supported, for example, by the association of macrofossils of the (now) upper subalpine-alpine zone mat conifer Microcachrys and the warm temperate genus Austromyrtus in Early to Middle Pleistocene deposits at Regatta Point, Strahan on the West Coast of Tasmania (Jordan et al. 1995, Macphail \& Hill 1995). For the same reason, we are unable to preclude the possibility that a lowland/cool-climate ecotype of Pherosphaera hookeriana was present in Tasmania during the earlier Pleistocene although no macrofossil evidence for this has been found to date. Despite this uncertainty, we conclude that the annual and seasonal rainfall at the time when Unit B was deposited was adequate to support a heterogeneous association of cool-cold temperate rainforest and rainforest scrub species including Nothofagus cunninghamii, and Phyllocladus, and sclerophyll taxa (pls 4, 5), but not rainforest per se, somewhere in the mountainous hinterland and/or on the coastal plain around Hannant Inlet.

\section{DISCUSSION}

The three microfloras preserved at Hannant Inlet are genetically linked in that these reflect different stages in the impact of Late Cenozoic global cooling (see fig. 2 in Zachos et al. 2008) on the Tasmanian flora and vegetation, a development that is "book-ended" by transient montane glaciation in northern Tasmania during the Early Oligocene (Macphail etal. 1993a) and periglacial and (mountain ranges) glacial environments across southern Tasmania during the Quaternary (see Colhoun etal. 2104). Notwithstanding the c. 23 million-year gap in the geologic record between Samples 1 and 3, the Hannant Inlet sequence is strategically sited to explore (a) phytogeographic links at high palaeolatitudes across the Southern Hemisphere and (b) climate-forced changes in coastal/lowland floras in South-West Tasmania preceding the Last Glacial Maximum at c. $22 \mathrm{ka}$.

\section{Phytogeographic links between South-West Tasmania, Antarctica and southern South America during the Late Paleogene}

The Oligocene microflora at Hannant Inlet postdates the submergence of the South Tasman Rise "landbridge" to Antarctica and onset of major ice-sheet glaciation in Antarctica during the Late Paleogene (see Exon et al. 2004, Truswell \& Macphail 2008). Although palynological dominants (and presumed canopy dominants) such as Araucariaceae, Podocarpaceae and Nothofagaceae, are common to East Antarctica, the South Tasman Rise, and Tasmania, this in likely to be an artefact of broad morphospecies definitions, i.e., the pollen types may represent different species or ecotypes. The same relationship is not reflected in the presumed subcanopy flora. For example, a number of the minor taxa recorded in East Antarctica and western Tasmania are common to both regions only during the Early Eocene when sea surface temperatures in Macquarie 
Harbour were at near tropical values (Pole \& Macphail 1996, Truswell 1997, Macphail 2002, 2007, Truswell \& Macphail 2008, Carpenter et al. 2012). Examples (NLRs in parentheses) are Polypodiaceoisporites (Pteris), Anacolosidites spp. (Anacolosa), Myrtaceidites tenuis (Eucalyptus sensu lato) and Intratriporopollenites notabilis (Tiliaceae).

This is not unexpected since most of these taxa are thermophiles that became extinct during the Middle Eocene, but a larger group of thermophiles found in Tasmania (and elsewhere across southern Australia) are recorded only as far south as the South Tasman Rise. Examples are Kuylisporites waterbolkii (Hemitelia), Verrucosisporites kopukuensis (Lygodium), Spinizonocolpites prominatus (Nypa), Beaupreaidites spp. (Beauprea) Cupanieidites orthoteichus (Sapindaceae tribe Cupanieae), Santalumidites cainozoicus (Santalaceae), Sapotaceoidaepollenites spp. (Santalaceae) and Bluffopollis scabratus (Strasburgeriaceae). A plausible corollary is that by the Middle-Late Eocene, the canopy as well as subcanopy floras had undergone significant adaptive radiation to local conditions and morphogenera apparently shared between southeastern mainland Australia (including Tasmania), Antarctica and South America represent different species or genera, as has been confirmed for the araucarian clade Dilwynites (Agathis/Wollemia) (Macphail et al. 2013). Contrary to expectation, e.g., Corbett (2014), the onset of cooler climates during the Eocene-Oligocene transition appears to have restricted the migration of cool-cold adapted species between Antarctica and Tasmania since two only morphospecies that evolved/migrated during this period can be confirmed to have appeared earlier in East Antarctica than in Tasmania. These are a Droseraceae (Fischeripollis sp.) whose closest NLR is the Venus-Flytrap genus Dionea now endemic to southern USA, and the extinct clade represented by Periporopollenites hexaporus. Significantly, specimens on both landmasses occur in glacial contexts (Macphail et al. 1993a, Macphail \& Hill 1994, Truswell \& Macphail 2008).

On the balance of probabilities, we conclude the majority of shared taxa migrated from Australia southwards onto the South Tasman Rise, and for the same reason, speculate that the reverse pathway, from East Antarctica to Tasmania via the South Tasman Rise, played only a minor role even during the benign conditions that characterised the Early Eocene hyperthermals (see Zachos et al. 2008). A reduced role for Antarctica in Cenozoic plant migration strengthens the case for trans-oceanic dispersal of plants across the palaeo-Pacific Ocean between Australia and southern South America. For example, at least three taxa found in the Early Oligocene glacigene sediments in Tasmania (two occur at Hannant Inlet) are not recorded in East Antarctica during the Late Eocene but are present in the Eocene-Oligocene in South America, viz. Lophosoria, Embothrium and Asteraceae tribe Mutisieae (fossil equivalent Mutisiapollis patersonii). In the case of Lophosoria, trans-oceanic dispersal between Australia and South America is strongly supported by the establishment of this groundfern on the Kergulen Islands in the remote southern Indian Ocean in the Miocene, as well as by occurrence of macrofossils of the sole extant South American species Lophosoria quadrapinnata (J.F.Gmelin)
C.Chr. in an Oligo-Miocene deposit at Balfour on the West Coast of Tasmania (Cookson 1947, Hill et al. 2001). Plant migration across the Pacific Ocean seems to have occurred in both directions since the earliest records of Lophosoria and Mutisieae occur in South America (Eocene) whilst Embothrium occur earlier in southeast Australia (Barreda et al., 2012, M. Quattrochio pers. comm. 2013).

\section{Early Late Pleistocene}

By the time Unit B was deposited in Hannant Inlet, the Tasmanian flora had been subjected to Quaternary "glacial winnowing" for up to $2.4 \mathrm{Ma}$ (see Macphail et al. 1993b, 1995). In this instance, the common occurrence of Pherosphaera and other cold-climate taxa at low elevations was expected because of (1) the inferred age of Unit B and (2) other fossil records of these taxa at low elevations during the Last and earlier Glacial Stages. For example, relict populations, macrofossils and/or pollen of Pherosphera hookeriana and other cold-climate taxa such as a plum pine, Podocarpus lawrencii Hook.f., the bolster-plant Donatia novae-zelandiae Hook.f. have been recorded at relatively low elevations in Tasmania, e.g., along the Douglas River on the East Coast, and in Penultimate Glacial Stage glacio-lacustrine clays underlying till near Rosebery (162 m elevation), in MiddleLate Quaternary lacustrine sediments infilling the Darwin Meteor Crater (180-120 m elevation) near the Andrew River and in the King River Valley (c. 160 m elevation) in western Tasmania (Macphail \& Moscal 1981, Colhoun \& van der Geer 1987, 1988, 1998, Gibson et al. 1987). With the apparent exception of the creeping pine, Microcachrys tetragona (Hook.f.) Hook.f., few modern cold-climate taxa in Tasmania are strictly "alpine" in that their populations now are confined to elevations above the climatic treeline. Nevertheless, as far as we are aware, the Hannant Inlet and Cox Bight sites are unique in preserving evidence that the Pherosphaera hookeriana actually grew close to the presentday coastline during the Late Pleistocene (cf. Bowman et al. 2013).

The extended range contrasts with the current distribution of Pherosphaera hookeriana. This shrub gymnosperm, together with the tree-fern Cyathea (Cyatheaceae) and at least two herbs, Gunnera (Gunneraceae) and Haloragodendrontype (Haloragaceae), form a small group of higher plants in Tasmania that have failed to recapture their pre-Last Glacial Maximum distribution (Colhoun 1980, M.K. Macphail unpubl. data). The reasons for this are unclear, especially since pollen data confirm that Pherosphera, as well as other cold-climate taxa not recorded at Hannant Inlet during the Late Pleistocene (Microcachrys, Astelia), had survived through several stadial/interstadial cycles at the low elevation Darwin Crater site. One possibility, which is supported by the delayed (late early Holocene) establishment of Pherosphaera in the subalpine zone on Mount Field (Macphail 1979), is its low vagility and competitive ability were inadequate to cope with rapid rises in mean air temperatures during the deglacial period and earlier Holocene period. However, this does not resolve the related question why other ecologically disparate taxa in the same 
group exhibited the same major contraction in range. An alternative working hypothesis is that the phenomenon is the result of the geologically recent extinction of unrecognised ecotypes as has been demonstrated for the genus Banksia (Jordan \& Hill 1991).

\section{CONCLUSIONS}

The Hannant Inlet microfloras are a significant geographic extension to what already has been recorded about the Cenozoic floras in Tasmania (Hill et al. 1999, Macphail et al. 2014). For example, fossil pollen of the subgenus (Lophozonia) of Nothofagus that includes $N$. cunninghamii, the modern dominant of cool temperate rainforest in Tasmania, are present in low but significant numbers (3-4\%) in OligoMiocene sequences at the Hannant Inlet and Fossil Bluff. However, the associate species differ markedly from those in extant cool temperate rainforest in Tasmania and the fossil record at Hannant Inlet is strongly against such forests being the lineal descendants of rainforests that occupied eastern Gondwana prior to its breakup in the Late Mesozoic-Early Paleogene (compare http://tarkine.org/rainforest/2014). Nevertheless, the wet-forest flora in South-West Tasmania is an ancient one, except at the species level, and extant families and some of the more prominent genera have been shared between Australia, Antarctica, New Zealand and southern South America for all or part of the Cenozoic. A surprising example is the eucalyptoid clade represented by Myrtaceidites tenuis, which occurs in Early Eocene deposits in Antarctica and southern South America as well as central and southern Australia, including Tasmania (Macphail et al. 1994, Hermsen et al. 2012, Thornhill \& Macphail 2012, Contrares et al. 2013, M.K. Macphail unpubl. data). The combined database confirms our belief that Tasmania preserves arguably the most geographically concentrated and often best-preserved natural archive in Australia of plant macrofossils and microfossils covering the last 65 million years (Cenozoic), as well as the only archive that covers the full altitudinal spectrum of environments over the Late Paleogene and Early Neogene (see Macphail 1987, Hill et al. 1999, Colhoun et al. 2014, Forsyth et al. 2014, Macphail et al. 2014). As for Hannant Inlet, the exploration of this "archive" is often dependent on chance discoveries. Any actions designed to promote the study of the geoheritage of this remote region can only be helpful in reconnecting Tasmania to its Gondwanan past.

\section{ACKNOWLEDGEMENTS}

We thank Quan Hua (ANSTO, Sydney) for providing the radiocarbon dates presented in table 2 and Associate Professor Greg Jordan (School of Plant Sciences, University of Tasmania) for analysis of a wood fragment from Unit B and his valuable comments on earlier drafts of this paper.

\section{REFERENCES}

Augustinus, P.C. \& Macphail, M.K. 1997: Early Pleistocene stratigraphy and timing of the Bulgobac Glaciation, western Tasmania. Palaeogeography, Palaeoclimatology, Palaeoecology 128: 253-267.

Barreda, V.D., Palazzesi1, L., Katinas, L., Crisci, J.V., Telleri, M.C., Bremers, K., Passala, M.G., Bechis, F. \& Corsolini, R. 2012: An extinct Eocene taxon of the daisy family (Asteraceae): evolutionary, ecological and biogeographical implications. Annals of Botany 109: 127-134.

Biffin, E, Brodribb, T.J., Hill, R.S., Thomas, P. \& Lowe, A.J. 2011: Leaf evolution in Southern Hemisphere conifers tracks the angiosperm ecological radiation. Proceedings of the Royal Society B [doi: 10.1098/rspb.2011.0559].

Bowman, D.M.J.S., Sharples, C., Hua, Q., Wood, S.W., Macphail, M.K. \& Haberle, S. 2013: Quaternary sub-fossil wood deposits at two locations in southwest Tasmania. Tasmanian Geological Survey Record 2013/01: $1-5$

Brown, M.J., Kirkpatrick, J.B. \& Moscal, A. 1983: An Atlas of Tasmania's Endemic Flora. Tasmania Conservation Trust, Hobart: 110 pp.

Carpenter, R.J., Jordan, J., Macphail, M.K. \& Hill, R.S. 2012: Near tropical Early Eocene terrestrial temperatures at the Austro-Antarctic margin. Geology 40: 267-270.

Colhoun, E.A. 1977: Late Quaternary fan gravels and slope deposits at Rocky Cape, northwestern Tasmania: their palaeoenvironmental significance. Papers and Proceedings of the Royal Society of Tasmania 111: 13-27.

Colhoun, E.A. 1980: Quaternary fluviatile deposits from the Pieman Dam site, western Tasmania. Proceedings of the Royal Society of London B207: 355-384.

Colhoun, E.A. \& van der Geer, G. 1987: Pleistocene macro- and micro- plant fossils from Rosebery, western Tasmania. Papers and Proceedings of the Royal Society of Tasmania 121: 89-92.

Colhoun, E.A. \& van der Geer, G. 1988: Darwin Crater, the King and Linda Valleys. In Colhoun, E.A. (comp). Cenozoic vegetation of Tasmania. Geography Department, University of Newcastle Special Paper (ISBN 072590621 9): 30-71.

Colhoun, E.A. \& van der Geer, G. 1998: Pollen analysis of 0-20 $\mathrm{m}$ at Darwin Crater, western Tasmania, Australia. In Horie, S. (ed). IPPCCE Newsletter 11: 68-89.

Colhoun, E.A., Harris, P.T., Heap, A., Bottrill, R.S., Bacon, C.A. \& Duncan, D. McP. 2014: The Quaternary in Tasmania. In Corbett, K.D., Quilty, P.G. \& Calver, C.R. (eds): Geological Evolution of Tasmania. Geological Society of Australia Special Publication 24, Geological Society of Australia (Tasmanian Division): 511-530.

Contreras, L., Pross, J., Bijl, P.K., Koutsodendris, A., Raine, J.I., van de Schootbrugge, B. \& Brinkhuis, H. 2013: Early to Middle Eocene vegetation dynamics at the Wilkes Land margin (Antarctica). Review of Palaeobotany and Palynology 197: 119-142.

Cookson, I.C. 1947: Plant microfossils from the lignites of the Kerguelen Archipelago. B.A.N.Z. Antarctic Research Expedition 1929-1931 Report Series A 2: 127-142.

Corbett, K.D. 2014: A summary of Tasmania's geology and geological history. In Corbett, K.D., Quilty, P.G. \& Calver, C.R. (eds): Geological Evolution of Tasmania. Geological Society of Australia Special Publication 24, Geological Society of Australia (Tasmanian Division): 1-12.

Exon, N., Kennett, J.P. \& Malone, M. (eds) 2004: The Cenozoic Southern Ocean: tectonics, Sedimentation and Climate Change between Australia and Antarctica. Geophysical Monograph 151: 1-367. 
Forsyth, S.M., Quilty, P.G. \& Calver, C.R. 2014: Cenozoic onshore basins and landscape evolution. In Corbett, K.D., Quilty, P.G. \& Calver, C.R. (eds) Geological Evolution of Tasmania. Geological Society of Australia Special Publication 24, Geological Society of Australia (Tasmanian Division): 437-450.

Gibson, N., Kiernan, K.A. \& Macphail, M.K. 1987: A fossil bolster plant from the King River, Tasmania. Papers and Proceedings of the Royal Society of Tasmania 121: 35-42.

Haq, B.U., Hardenbol, J. \& Vail, P. 1987: Chronology of fluctuating sea levels since the Triassic. Science 235: 1156-1167.

Hermsen, E.J., Gandolfo, M.A. \& Zamaloa, M. del C. 2012: The fossil record of Eucalyptus in Patagonia. American Journal of Botany 99: 1356-1374.

Hill, R.S., Macphail, M.K. \& Jordan, G.J. 2001: Macrofossils associated with the fossil fern spore Cyatheacidites annulatus and their significance for Southern hemisphere biogeography. Review of Palaeobotany and Palynology 116: 195-202.

Hill, R.S., Macphail, M.K. \& Jordan, G.J. 1999: Tertiary history and origins of the flora and vegetation. In Reid, J.B., Hill, R.S., Brown, M.J. \& Hovenden, M.J. (eds). Vegetation of Tasmania. Flora of Australia Supplementary Series Number 8, Australian Biological Resources Study, Tasmania: 39-63.

Houben, A.J.P., van Mourik, C.A.\& Montanari, A., Coccioni, R. \& Brinkhuis, H. 2012: The Eocene-Oligocene transition: Changes in sea level, temperature or both? Palaeogeography, Palaeoclimatology, Palaeoecology 335-336: 75-83.

Houshold, I. Chappell, J. \& Fifield, K. 2006: Geomorphic response to intraplate neotectonics - Birch's Inlet, SW Tasmania. ANZGG Proceedings (Meeting No. 12, Taipa Bay, NZ 2006).

Jordan, G. \& Hill, R.S. 1991: Two new Banksia species from Pleistocene sediments in western Tasmania. Australian Systematic Botany 4: 499-511.

Jordan, G.J., Carpenter, R.J. \& Hill, R.S. 1991: Late Pleistocene vegetation and climate near Melaleuca Inlet, south-west Tasmania, as inferred from fossil evidence. Australian Journal of Botany 39: 315-333.

Jordan, G.J., Barnes, R., Hill, R.S. \& Macphail, M.K. 1995: An Early to Middle Pleistocene flora of subalpine affinities in lowland western Tasmania. Australian Journal of Botany 43: 231-242.

Lewis, D. \& Quilty, P.G. 2009: Foraminifera and palaeoenvironment of elevated Late Pleistocene sands, White Rock Point, southeastern Tasmania. Papers and Proceedings of the Royal Society of Tasmania 143: 95-100.

Macphail, M.K. 1979: Vegetation and climates in Southern Tasmania since the last glaciation. Quaternary Research 11: $306-341$

Macphail, M.K.1987: Palynological Resources of the Lemonthyme and Southern Forests Protected Areas. Consultancy Report 134A. In Report of the Commission of Inquiry into the Lemonthyme and Southern Forests 1: 147-148, 152-156, Australian Government Publishing Service, ACT.

Macphail, M.K. 1996: A habitat for the enigmatic Wynyardia bassiana Spencer 1901, Australia's first described Tertiary land mammal? Alcheringa 20: 227-243.

Macphail, M.K. 1999: Palynostratigraphy of the Murray Basin, inland southeast Australia. Palynology 23: 199-242.

Macphail, M.K. 2002: ODP leg 189: Initial results: terrestrial plant microfossils. Ocean Drilling Program/Australian Geological Survey Organisation, 34 pp. + Appendices.

Macphail, M.K. 2007: Australian Palaeoclimates Cretaceous to Tertiary. A review of palaeobotanical and related evidence to the year 2000. CRC-LEME Open File Report 151 (Special Volume ISBN 192103975 2) 279 pp.

Macphail, M.K. \& Moscal, A. 1981: Podocarpus lawrencii (Hook f. in Hook) on the East Coast: relict from last glacial times? Papers and Proceedings of the Royal Society of Tasmania 115: 1-3.

Macphail, M.K. \& Hill, R.S. 1994: K-Ar dated palynofloras in Tasmania 1: Early Oligocene Proteacidites tuberculatus Zone sediments, Wilmot Dam, northwestern Tasmania. Papers and Proceedings of the Royal Society of Tasmania 128: 1-15.

Macphail, M.K. \& Hill, R.S. 1995: A fossil flora from rafted Plio-Pleistocene mudstones at Regatta Point, Tasmania. Australian Journal of Botany 33: 497-517.

Macphail, M.K., Colhoun, E.A., Kiernan, K.W. \& Hannan, D. 1993a: Glacial climates in the Antarctic region during the late Paleogene: evidence from North-West Tasmania. Geology 21: 145-148.

Macphail, M.K., Jordan, G.J. \& Hill, R.S. 1993b: Key periods in the evolution of the Cenozoic vegetation and flora in western Tasmania 1: the Early-Middle Pleistocene. Australian Journal of Botany 41: 673-707.

Macphail, M.K., Alley, N., Truswell, E.M. \& Sluiter, I.R. 1994: Early Tertiary vegetation: evidence from pollen and spores. In Hill, R.S. (ed) Australian Vegetation History: Cretaceous to Recent, Cambridge University Press, Cambridge: 189-261.

Macphail, M.K., Colhoun, E.A. \& Fitzsimons, S.J. 1995: Key periods in the evolution of the Cenozoic vegetation and flora in western Tasmania: the Late Pliocene. Australian Journal of Botany 43: 505-526.

Macphail, M.K., Pemberton, M. \& Jacobson, G. 1999: Peat mounds of southwest Tasmania: possible origins. Australian Journal of Earth Sciences 46: 667-677.

Macphail, M., Carpenter, R.J., Iglesias, A. \& Wilf, P. 2013: First evidence for Wollemi Pine-type pollen (Dilwynites: Araucariaceae) in South America. PLoS ONE 8(7): e69281 [doi:10.1371/journal.pone.0069281].

Macphail, M.K., Hill, R.S., Partridge, A.D. \& Jordan, G.J. 2014: Geo-botany of the Cretaceous to Neogene. In Corbett, K.D., Quilty, P.G. \& Calver, C.R. (eds) Geological Evolution of Tasmania. Geological Society of Australia Special Publication 24, Geological Society of Australia (Tasmanian Division): 495-507.

Miller, K.G., Mountain, G.S., Leg 150 Shipboard Party, \& members of the New Jersey coastal plain drilling project. 1996: Drilling and dating New Jersey Oligocene-Miocene sequences: ice volumes, global sea levels and Exxon records. Science 271: 1092-1095.

Ogg, J.G., Ogg, G. \& Gradstein, F.M. 2008: The Concise Geologic Time Scale. Cambridge University Press, Cambridge: $177 \mathrm{pp}$.

Parfitt, S.A. \& 15 co-authors 2010: Early Pleistocene occupation at the edge of the boreal zone in northwest Europe. Nature 466: 229-233.

Parks and Wildlife Service, Tasmania 2003: Melaleuca-Port Davey Area Plan 2003. Department of Tourism, Heritage and the Arts, Hobart: 94 pp.

Partridge, A.D. 1999: Late Cretaceous to Tertiary Geological Evolution of the Gippsland Basin, Victoria. 2 Volumes. Unpublished PhD Thesis, Latrobe University, Melbourne: 439 pp.

Partridge, A.D. 2006: Late Cretaceous-Cenozoic palynology zonations, Gippsland Basin. In Monteil, E. (coord.) Australian Mesozoic and Cenozoic Palynology Zonations - updated to the 2004 Geologic Time Scale. Geoscience Australia Record 2006/23 [ISBN I 92123605 1].

Pole, M. \& Macphail, M.K. 1996: Eocene Nypa from Regatta Point, Tasmania. Review of Palaeobotany and Palynology 92: 55-67.

Quilty, P.G. 1972: The biostratigraphy of the Tasmanian marine Tertiary. Papers and Proceedings of the Royal Society of Tasmania 106: 25-44.

Quilty, P.G. 2014: Onshore Neogene marine sediments. In Corbett, K.D., Quilty, P.G. \& Calver, C.R. (eds) Geological Evolution of Tasmania. Geological Society of Australia 
Special Publication 24, Geological Society of Australia (Tasmanian Division): 450-451.

Quilty, P.G. \& Seymour, D.B. 2010: Early Miocene silicified limestone From Temma, Northwestern Tasmania: Further evidence of substantial post-early Miocene uplift or tilting of Tasmania Papers and Proceedings of the Royal Society of Tasmania 144: 43-50.

Read, J. \& Hope, G.S. 1996: Ecology of Nothofagus forests of New Guinea and New Caledonia. In Veblen, T.T., Hill, R.S. \& Read, J. (eds). The Ecology and Biogeography of Nothofagus Forests. Yale University Press, New Haven: 200-256.

Sims, P.C. 2013: No reprieve for Tasmanian rock art. Arts 2: $182-224$.

Stefanski, M.Z. 1957: Progress report on regional geological survey of the Port Davey-Cox Bight area. Tasmanian Department of Mines Technical Report 2: 87-106.

Thomas, I. 1995: Where has all the forest gone; new pollen evidence from Melaleuca Inlet, SW Tasmania. Monash University Publications in Geography 45: 295-301.

Thornhill, A. \& Macphail, M.K. 2012: Fossil myrtaceous pollen as evidence for the evolution of the Myrtaceae: A review of fossil Myrtaceidites species. Review of Palaeobotany and Palynology 176-177: 1-23.

Toghill, P. 2000: The Geology of Britain: an introduction. Airlife Publishing, Marlborough. 192 pp.

Traverse, A. 1988: Palaeopalynology. Unwin Hyman, Boston: $600 \mathrm{pp}$.

Truswell, E.M. 1997: Palynomorph assemblages from marine Eocene sediments on the west Tasmanian continental margin and the South Tasman Rise. Australian Journal of Earth Sciences 44: 633-654.

Truswell, E.M. \& Macphail, M.K. 2008: Polar forests on the edge of extinction: what does the fossil spore and pollen evidence from East Antarctica say? Australian Systematic Botany 22: 57-106.

Tulip, J.R., Taylor, G. \& Truswell, E.M. 1982: Palynology of Tertiary Lake Bunyan, Cooma, New South Wales. BMR Journal of Australian Geology and Geophysics 7: 255-268.

Veevers, J.J., Powell, C. McA. \& Roots, S.R. 1991: Review of seafloor spreading around Australia. 1. Synthesis of the patterns of spreading. Australian Journal of Earth Sciences 38: 373-389.

Williams, P.R. 1981: Stratigraphic terminology of low-grade metamorphosed rocks at Bathurst Harbour, S.W. Tasmania. Papers and Proceedings of the Royal Society of Tasmania 115: 21-28.

Williams, P.R. 1982: Davey, Tasmania. Tasmanian Department of Mines Geological Atlas 1: 50000 Series Explanatory Report, Sheet 91 (8011S).

Williams, P.R. \& Corbett, E.B. 1977: Geological Atlas 1: 250 000 Series Sheet SK-55/7. Port Davey. Explanatory Report of the Geological Survey of Tasmania, Hobart: 14 pp.

Wood, S.W., Hua, Q. \& Bowman, D.M.J.S. 2011a: Fire-patterned vegetation and the development of organic soils in the lowland vegetation mosaics of south-west Tasmania. Australian Journal of Botany 59: 126-136.

Wood, S.W., Murphy, B.P. \& Bowman, D.M.J.S. 2011 b: Firescape ecology: how topography determines the contrasting distribution of fire and rain forest in the south-west of the Tasmanian Wilderness World Heritage Area. Journal of Biogeography 38-39: 1807-1820.

Zachos, J.C., Dickens, G.R. \& Zeebe, R.E. 2008: An early Cenozoic perspective on greenhouse warming and carboncycle dynamics. Nature 451: 279-283.

(accepted 2 December 2014) 
